\section{Variable noise and information transmission}

\author{
RICHARD V. ALUMBAUGH and KENICHI TAKEMURA \\ Central Washington State College, Ellensburg, Wash. 98926
}

Noise levels were varied systematically to determine the effects on information processing. Slides consisting of circular dots (relevant information) and triangles (irrelevant information) were presented tachistoscopically. The task of Ss was to correctly identify the number of dots; dots varied from 3 to 12 quantitatively, while triangles varied from 0 to 3 . The results suggested that (1) noise may have a limen similar to channel capacity for relevant stimuli and (2) response set needs to be examined more closely as a biasing effect on information transmission.

Few studies have investigated the effects of varying noise levels while presenting variable information loads. Studies investigating the "span of apprehension" have shown that humans can accurately process about eight units (Woodworth \& Schlosberg, 1954). Such a limitation has been hypothesized by Miller (1956) to exist for all other sensory modalities.

Visual masking tangentially relates to the effects of noise information processing (Kahneman, 1968). Visual masking, which refers to the effects of a given masking stimulus (MS) on a test stimulus (TS), has generally been limited to the temporal contiguity situation. The MS may temporally precede or follow the TS, and the effect on information transmission is measured. Masking by visual noise has been found to be more effective than light (Schiller \& Wiener, 1963; Sperling, 1963; Schiller, 1965; Scharf, Zamanski, \& Brightbill, 1966; Smith \& Schiller, 1966). Most investigators concur that masking by noise (pattern) is most critical when the interstimulus interval (ISI) between TS and MS is zero.

With an ISI of zero, a recent study (Alumbaugh, 1968) has shown that information processing monotonically decreases as a function of noise level for psychiatric and community populations. The present study expanded both the levels of noise and information. The purpose ofthe study was to investigate more precisely how noise interacts with a span of apprehension using a college population.

\section{SUBJECTS}

Seventy-five undergraduate males and 45 undergraduate females enrolled in a psychology of adjustment course were selected randomly from a pool of volunteers. Each $\mathbf{S}$ was given course credit for participation in the study. All were given pretrials of the experimental task to eliminate those that experienced visual difficulties with the apparatus.

$$
\text { APPARATUS }
$$

A Lehigh Valley Model 111-10 tachistoscope was mounted on a 1 x $10 \times 8$ in. plank and affixed to a table noise. at the height of 38 in. from the floor. A hooded $12 \times 14$ in. translucent screen was mounted on the plank opposite the projector. The tachistoscope was set for $.50 \mathrm{sec}$ exposure. Slides were presented via a microswitch accessible to the Ss. The apparatus was placed in a soundproofed room to minimize the extraneous auditory noise of relays.

Slides were constructed with the use of a 12 by 12 matrix and a random numbers table. Black-filled 1/4-in.-diam circles were assigned randomly to the matrix via a set of random number coordinates. Similarly, equilateral triangles with a $1 / 2$-in. base were placed on the matrix with the use of random numbers.

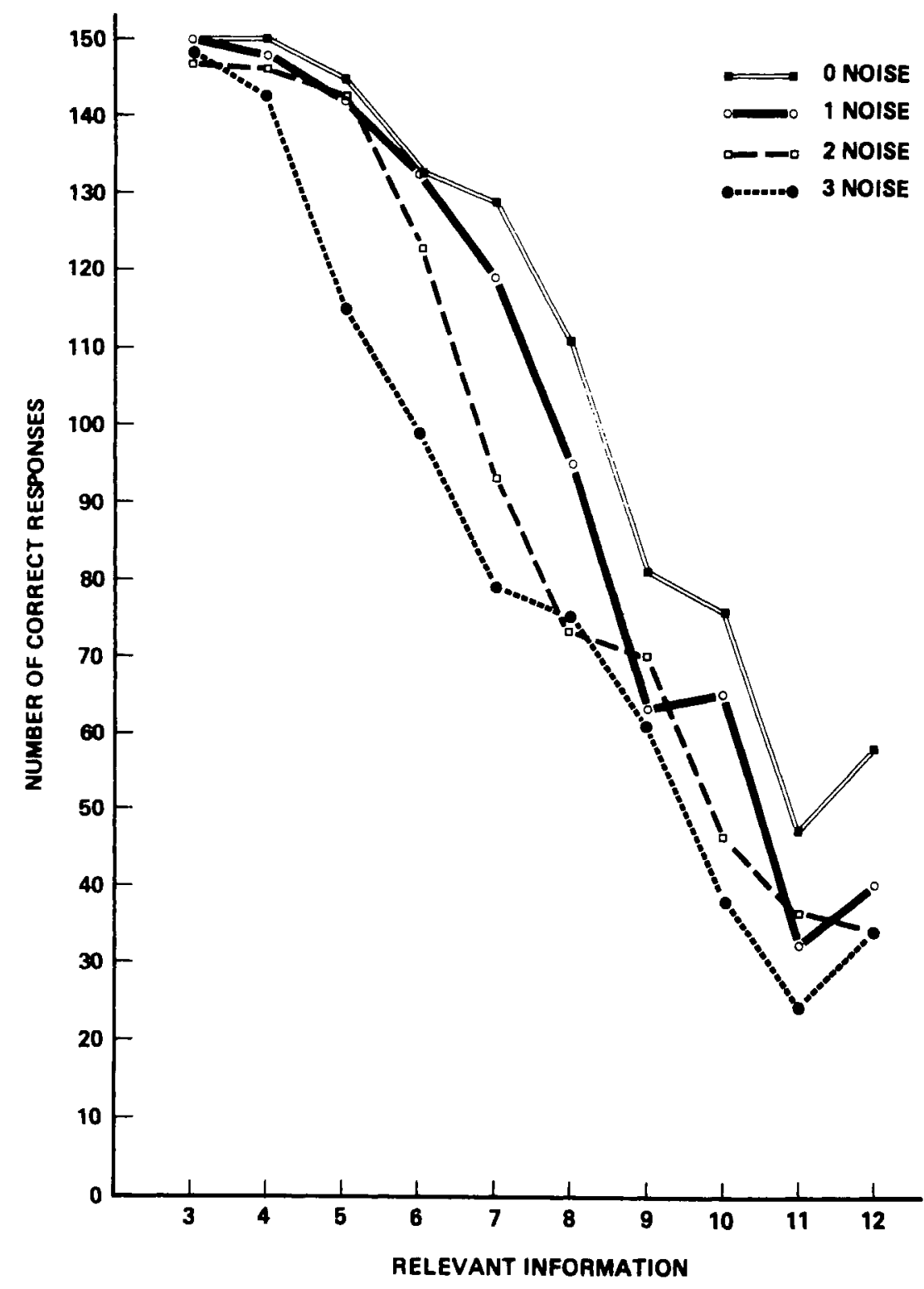

Fig. 1. Mean number correct across information load for each level of 


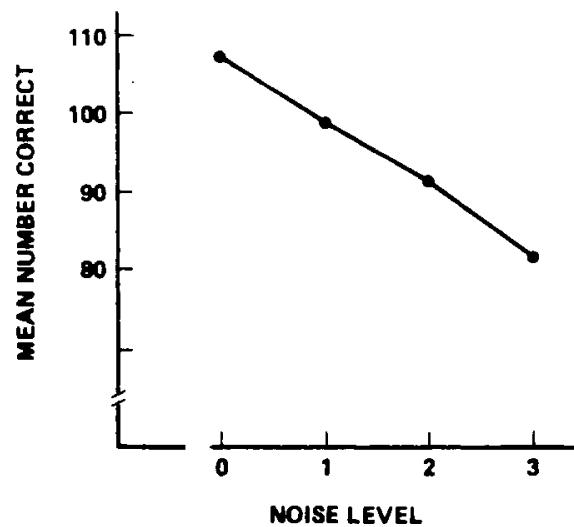

Fig. 2. A comparison of the total number correct for each of the noise groups across the 10 levels of information.

\section{PROCEDURE}

The Ss were assigned randomly to one of four noise conditions, ranging from zero to three. Each $S$ was instructed to fix his eyes to the screen and to push the stimulus switch to active the slide projector. The instructions were simply to count the number of filled circles or dots that appeared on the screen. No reference was made to the triangles or visual noise. Ss recorded their responses on answer sheets.

Stimuli were arranged such that the filled circles (information), ranging from 3 to 12, appeared in a field of triangles, ranging from 0 to 3 . Ss who were assigned to a given noise level were exposed to 50 slides, or five presentations of each of the 10 levels of information.

Six pretrials were presented, using either one or two filled circles with no triangles presented. Ss were excluded for failure on any of these trials. All trials were S-paced, and the E left the room once the pretrials had been administered.

\section{RESULTS}

A split-plot (Kirk, 1969) analysis of variance was applied to the 10 nested levels of information for each of the four noise groups. The two treatments of noise and information were both significant beyond the .01 level ( $\mathrm{df}=$ $3 / 116$ and 9/1044). The Noise by Information interaction was significant at the .01 level $(\mathrm{df}=27 / 1044)$.

Since the Information by Noise interaction was significant, a simple main effects analysis was conducted to determine at what levels noise and information interacted. The analysis for information revealed that Levels 5 and 6 were significant at the .05 level ( $\mathrm{df}=3 / 27$ ), while Levels 7,8 , and 10 were significant at the .01 level ( $\mathrm{df}=$ $3 / 27$ ). No significant interaction effects were found with Levels $3,4,9$, 11 , or 12. A simple main effects analysis for the four noise conditions showed that all four noise levels were associated with significant interaction effects ( $d f=9 / 1044, p<.01)$.

Mean differences for the four noise levels (Fig. 1) were tested with Tukey's HSD test. Comparing all possible combinations of means, differences were found to be significant beyond the .01 level with $\mathrm{df}=116$. A similar comparison was made for given levels of information load. Figure 2 shows that the mean scores decrease at Level 4 with increasing information. The exception to this trend is at Stimulus Level 12 . Since Levels 11 and 12 were not associated with interaction effects, a post hoc comparison was made using Tukey's posterior test. Comparing Level 11 with Level $12, \mathrm{~F}=34.039$, df $=10 / 1044, \mathrm{p}<.01$.

\section{DISCUSSION}

The study confirms that levels of information and varying levels of noise interact with the ISI held at constant zero. The significant interaction effects occur in the range of channel capacity, as reported by Miller (1956), i.e., 5 to 8 units. Apparently, increasing noise interferes with successful transmission at lower information loads. Such an effect may be due to discrimination problems brought on by high information loads. Therefore, it would be expected that performance would decrease at higher noise levels as a function of increasing information.

Though such a decrease was found at higher noise and information levels, there were no significant interaction effects at levels higher than 8 units, other than Level 10. It is conjectured that "approximating information" replaces subitizing (Miller, 1956) at higher information levels. The $S$ is no longer perceiving precise elements, but rather, there is a tendency toward chunking information. Noise at these levels reduces accuracy, but it does not diminish performance to the degree of subitizing high noise and moderate information loads.

The curious increase in performance at Information Level 12 can be explained partially by approximation responses. The number 12 has special significance in this culture; various measures, such as the foot and dozen, are frequently used as unit measures. Subsequently, the uncertainty of high noise and information loads may have induced Ss to make more accurate 12 responses to information loads in this range. Such a finding suggests that future research concentrate on the influence of other approximation responses that are culturally emphasized.

\section{SUMMARY}

Ss were tachistoscopically presented with varying combinations of information and noise. The results confirmed that noise interacts with information load. Such an interaction occurs in the range of channel capacity, as discussed by Miller. Higher information and higher noise-level combinations suggest that Ss switch from subitizing responses to a p poximation responses. Approximations seem to be influenced by cultural sets, since 12 units of information at all noise levels showed an increase in performance. Cultural set for number should be examined in other investigations where high information or noise loads are considered.

\section{REFERENCES}

ALUMBAUGH, R. V. The relationship between relevant and irrelevant information in chronic schizophrenia. Unpublished doctoral dissertation, Texas Tech University, 1968 .

KAHNEMAN, D. Method, findings, and theory in studies of visual masking. Psychological Bulletin, 1968. 69 , 218-226.

KIRK, R. E. Experimental design: Procedures for the behavioral sciences. Belmont, Calif: Brooks/Cole, 1969

MILLER, G. A The magical number seven, plus or minus two: Some limits on our capacity for processing information. Psychological Review. 1956, 63, 81-97.

SCHARF, B., ZAMANSKI, H, S., \& BRIGHTBILL, R. F. Word recognition with masking. Perception \& Psychophysics, 1966, 1, 110-112.

SCHILLER, P. H. Monoptic and dichoptic visual masking by patterns and flashes. Journal of Experimental Psychology, $1965,69,193-199$.

SCHILLER, P. H., \& WIENER, M Monoptic and dichoptic visual masking. Journal of Experimental Psychology, $1963,66,386-393$.

SMITH, M. C., \& SCHILLER, P. H. Forward and backward masking: A comparison. Canadian Journal of Psychology, 1966, 20. 191-197.

SPERLING, G. A model for visual memory tasks. Human Factors, 1963, 5, 19-31.

WOODWORTH. R. S., \& SCHLOSBERG, H. Experimental psychology. New York: Holt, Rinehart \& Winston, 1954. Pp. 90-105. 